\title{
DESARROLLO DE LA ICTIOLOGIA: EXTENSION HACIA LA ENSEÑANZA BASICA Y MEDIA EN CHILE
}

\author{
ICHTHYOLOGY DEVELOPMENT: EXTENSION TO ELEMENTARY AND \\ HIGH SCHOOL TEACHING IN CHILE
}

\author{
Laura G. Huaquín \\ Facultad de Ciencias Veterinarias y Pecuarias, Universidad de Chile. Casilla 2 Correo 15, Santiago. \\ Email: lhuaquin@uchile.cl
}

\begin{abstract}
RESUMEN
Con el objeto de hacer extensivos los conocimientos ictiológicos e incrementar las posibilidades de desarrollo de esta disciplina en nuestro país, se propone la creación de un Programa Educativo a Distancia para la enseñanza de la Ictiología, considerando el desarrollo de temas básicos y especializados, al alcance de enseñanza básica y media, con la finalidad de motivar en estos estudiantes las inquietudes hacia el conocimiento de la fauna íctica tanto la representada en los ambientes marinos como dulceacuícolas del país. El diagnóstico sobre el conocimiento de este tipo de fauna en el ciudadano común es bastante precario. Chile es un país con grandes recursos hidrológicos, una extensa costa, numerosas cuencas, más de 1.000 especies de peces marinos descritas y una fauna íctica dulceacuícola empobrecida. Sin embargo, la población en general, incluso profesionales desconocen la temática, lo que incide en las decisiones de altos niveles administrativos. Esta es una formación que debe comenzar desde la enseñanza básica. Es necesario hacer un esfuerzo para incentivar proposiciones en las que estén presentes estas temáticas.

El tema presentado en el VII Simposio-Taller de Ictiología, a modo de pregunta al escolar: “¿Conoces los peces de Aguas Continentales de Chile?", conformada en una clase desarrollada para estudiantes de $7^{\circ}$ y $8^{\circ}$ años, podría plantearse como extensión y a modo de ejemplo, en la educación extraescolar o en talleres de ciencias. Propongo a la Asociación de Ictiología de Chile liderar un Programa Nacional de Enseñanza de estos tópicos en el país.
\end{abstract}

Palabras Claves: Programa Educativo, ictiología, enseñanza básica y media, Chile.

\section{ABSTRACT}

A proposal is made to develop basic and specialized topics in ichthyology for a Distance Learning Program for elementary and high school students in Chile. The extension of ichthyological knowledge towards high school education will help to increase the development possibilities of this discipline in Chile. Hopefully this Program may be able to stimulate the Ministry of Education to promote interest towards Chilean marine and freshwater ichthyofauna in such students. The diagnosis about the knowledge that the common citizen has of this type of fauna is quite insufficient. Although Chile is a country with great hydrological resources, an extensive coast, more than 1000 described marine fish species, many hydrological watersheds, and, unfortunately, an impoverished freshwater fish fauna, the population, as a whole, and even professionals, are unaware of this issue, which impinges on high level decisions. This is a formation that should start from elementary education. An attempt to promote proposals involving the ichthyological topics is necessary. The topic “ ¿Know Continental Freshwater Fishes of Chile?”, developed for $7^{\text {th }}$ and $8^{\text {th }}$ grade students, is presented as an extension activity towards extracurricular education or workshops in sciences. I propose that the Chilean Ichthyological Association should lead a National Program of Ichthyology teaching for improving this education topic in the country.

KeYwords: Educational Program, Ichthyology, Elementary and High School Education. 
Ictiología en la enseñanza básica y media en Chile: HuAQuín, L.

\section{INTRODUCCION}

\section{LA NECESIDAD DE CONOCIMIENTO Y FORMACIÓN}

La falta de educación, conocimientos, aprendizaje y formación de amplios segmentos de la población han afectado el crecimiento de los países en América Latina. Esta es una gran necesidad actual, tenemos la materia prima pero nos ha faltado el desarrollo intelectual más democrático, pieza clave que amplía la visión del mundo contemporáneo. La brecha será más honda si se mantienen estratos educacionales sociales y si no captamos la esencia de nuestras diferencias, las cuales en el mundo globalizado pueden entrar a sobresalir y nos permitirán cambios que mejoren nuestra posición.

Cada región, cada país tiene peculiaridades destacables que, apreciándolas o no, son de por sí materias importantes de conocimientos, forman un conjunto de tópicos propios con un sello o impronta especial. Así como ejemplos concretos, sin desmerecer otros, no podemos desconocer en Ecuador su fauna típica, reptiles y aves de las islas Galápagos. En Bolivia, las alturas altiplánicas, el Lago Titicaca y su invierno boliviano. Argentina y sus pampas, sus estancias y gran producción ganadera. El cobre por un lado, el mar presencial de Chile y sus especies continentales e insulares endémicas, entre otros. Los recursos marítimos en Chile han sido una fuente importante de sustento, entre ellos los peces corresponden a los productos de mayores desembarques tanto de la pesquería artesanal como industrial. Pequeño (1989) hace una lista sistemática revisada y comentada de los peces de Chile. Ruiz y Marchant (2004), elaboraron un completo texto docente sobre la ictiofauna de aguas continentales chilenas, comentando su estado de conservación. Desde hace más de 50 años que se ha venido constatando una disminución importante en la cantidad de especies ícticas continentales nativas y en sus poblaciones (Huaquín y Manríquez 1986). Vila et al. (1999) señalan que los peces límnicos de Chile poseen caracteres especiales y muy diferentes de los peces de otras regiones del mundo, como son, su tamaño pequeño, alto grado de endemismo y baja riqueza de especies. En el caso de los peces marinos, la observación de las estadísticas de los últimos 6 años arroja una disminución en las cantidades desembarcadas respecto a la década anterior (SERNAPESCA 2002). Estos antecedentes, poco difundidos, son información necesaria para la docencia básica y media.

En Chile se ha hecho un esfuerzo notable en materias educacionales durante los últimos años; se están revisando planes y programas. Aprovechando esta oportunidad sería necesario implementar en ellos aspectos destacables de nuestra identidad como país, de los recursos biológicos y muy especialmente por la situación geográfica de país costero, de los recursos hidrobiológicos como los ictiológicos, malacológicos, carcinológicos y otros. El diagnóstico sobre el conocimiento de este tipo de fauna en el ciudadano común es bastante precario y desalentador. Ahora en que la calidad misma de la educación se encuentra abierta a críticas por la reforma en sus distintos niveles, parece interesante plantear esta proposición con la finalidad de dar refuerzo tanto a los profesores de niveles básico y medio como a los diferentes estudiantes en estos tópicos.

\section{LA ICTIOLOGÍA}

El verdadero desarrollo de una ciencia como la ictiología tiene su base sobre una fuerte investigación, aspecto que conlleva a la especialización cada vez más acelerada y divisoria del conocimiento. Actualmente podemos separarla en campos o especialidades diversos, los ictiólogos moleculares tienen sus propias metodologías y búsquedas de información en forma diferente a los ictiólogos dedicados a la fisiología o a los patólogos de peces e incluso a los anatomo-morfólogos y a los taxónomos. Los objetivos transversales se han transformado ahora en una verdadera necesidad del conocimiento enfrentado a la profunda especialización. Es así como los opuestos tienden a juntarse nuevamente. Un concepto integrador es el de la diversidad y para su análisis requiere que entren en juego todas estas especialidades. La ictiología entonces podría fortalecerse al ser enfocada desde una perspectiva global y multidisciplinaria a la vez, conformada por aspectos moleculares, genéticos, pasando por los anatomo-morfológicos hasta los cladísticos, etológicos y ecológicos; estas relaciones múltiples y con otras disciplinas constituyen los objetivos transversales

¿Qué son los objetivos transversales en educación?: son sencillamente los nexos que pueden relacionar una disciplina con otra. 
Los programas en que se trata la ictiología actualmente se imparten casi exclusivamente en carreras de acuicultura, biología marina, biología pesquera y en los postgrado de la temática obviamente. En la mayoría de las carreras del área biológica (pregrado) el tema se trata en su mayor parte en los cursos de zoología de vertebrados, como es el caso de las ciencias veterinarias. No existe la asignatura como tal, menos aún se imparte este conocimiento en otras carreras más alejadas como son la ingeniería en recursos naturales o las de biotecnología y otras que han aparecido últimamente. En la educación media y en la educación básica es en la práctica, un conocimiento olvidado. A menudo se cree tener algún conocimiento por tener información sobre fauna íctica exótica que es sólo cultivada en el país.

Sin embargo hay un conjunto de profesionales (Ingenieros en recursos, biotecnólogos, médicos Veterinarios, e incluso abogados y economistas) que deberían entender aspectos básicos de la ictiología, con el fin de tener la información necesaria para analizar los estudios de impacto, considerar las relaciones de los procesos que involucran a los recursos naturales y tomar las decisiones informadas. Estas premisas, que valdrían para todos los recursos, las estoy planteando en relación con la información ictiológica, pues nos encontramos en un punto en el cual la valoración de estos recursos nativos marinos, estuariales o de aguas continentales es escasa. En la formación de los profesores hasta ahora la ictiología o los recursos hidrobiológicos no se cuentan entre las materias de sus programas.

Por otro lado sería deseable que esta disciplina fuera también parte de los programas de enseñanza básica media incluidos en la temática ambiental, o de ciencias, tanto en cursos regulares como electivos, abarcando la educación extraescolar. Un país con más de 4.000 kilómetros de borde costero amerita que sus habitantes tengan una formación importante en estos tópicos.

EDUCACIÓN PARA LA CONSERVACIÓN DE LA DIVERSIDAD Y EL MEDIO AMBIENTE

Se han planteado diversas propuestas sobre educación para la conservación, educación del medio rural (Astudillo \& López 1987), educación ambiental para comunidades ribereñas (López \& Monsalve 1994), métodos para la conservación de la biodiversidad (Burbidge 1995) y la urgencia de consolidar la ciencia de la biodiversidad como una disciplina integradora (Cracraft 1995). Por otro lado, para elevar el nivel de competencia habría que iniciar tempranamente estos temas, ya que como hacen ver Young et al. (2002) "en los comienzos del siglo 21 ninguna educación puede considerarse adecuada en los países en desarrollo sin un amplio componente científico". Si consideramos que es parte de nuestra responsabilidad el que los temas ictiológicos estén presentes en los programas educacionales, debemos hacer un esfuerzo para incentivar proposiciones y estar presentes con estas temáticas en los diferentes niveles señalados. Específicamente se requiere promover y reforzar estos conocimientos hacia los profesores de enseñanza básica y media, que son directos responsables de propiciar y motivar el cambio educativo en sus estudiantes.

El Decreto 40 del 24 de enero de 1996 establece los objetivos fundamentales y contenidos mínimos obligatorios para la educación básica chilena fijando normas para su aplicación. En relación con sus objetivos persona-entorno, en este proceso de socialización escolar se debe desarrollar en los alumnos capacidades "para proteger el entorno natural y promover sus recursos como contexto de desarrollo humano" y, además "reconocer y valorar las bases de la identidad nacional en un mundo cada vez más globalizado e independiente" (Ministerio de Educación 1996). Sobre esta amplia base pueden integrarse las temáticas sobre recursos hidrobiológicos.

Las facilidades que entrega la educación a distancia y las destrezas alcanzadas en el uso de mapas conceptuales (Novak 1990), que son una herramienta interesante para el aprendizaje significativo y que representan ideas y conceptos claves enlazados junto a proposiciones en una estructura que facilita la visualización para el aprendizaje (Fig. 1), pueden ayudar en forma importante tanto a la organización como al uso del conocimiento; estas son herramientas útiles a los propósitos aquí planteados. La Asociación Chilena de Ictiología podría perfectamente liderar un Programa Nacional de enseñanza de la Ictiología en Chile, con la participación de todos los que comparten estas inquietudes. Existen buenos y calificados investigadores en las diversas especialidades de la Ictiología con publicaciones en revistas nacionales y del extranjero. Sería largo 
Ictiología en la enseñanza básica y media en Chile: Huaquín, L.
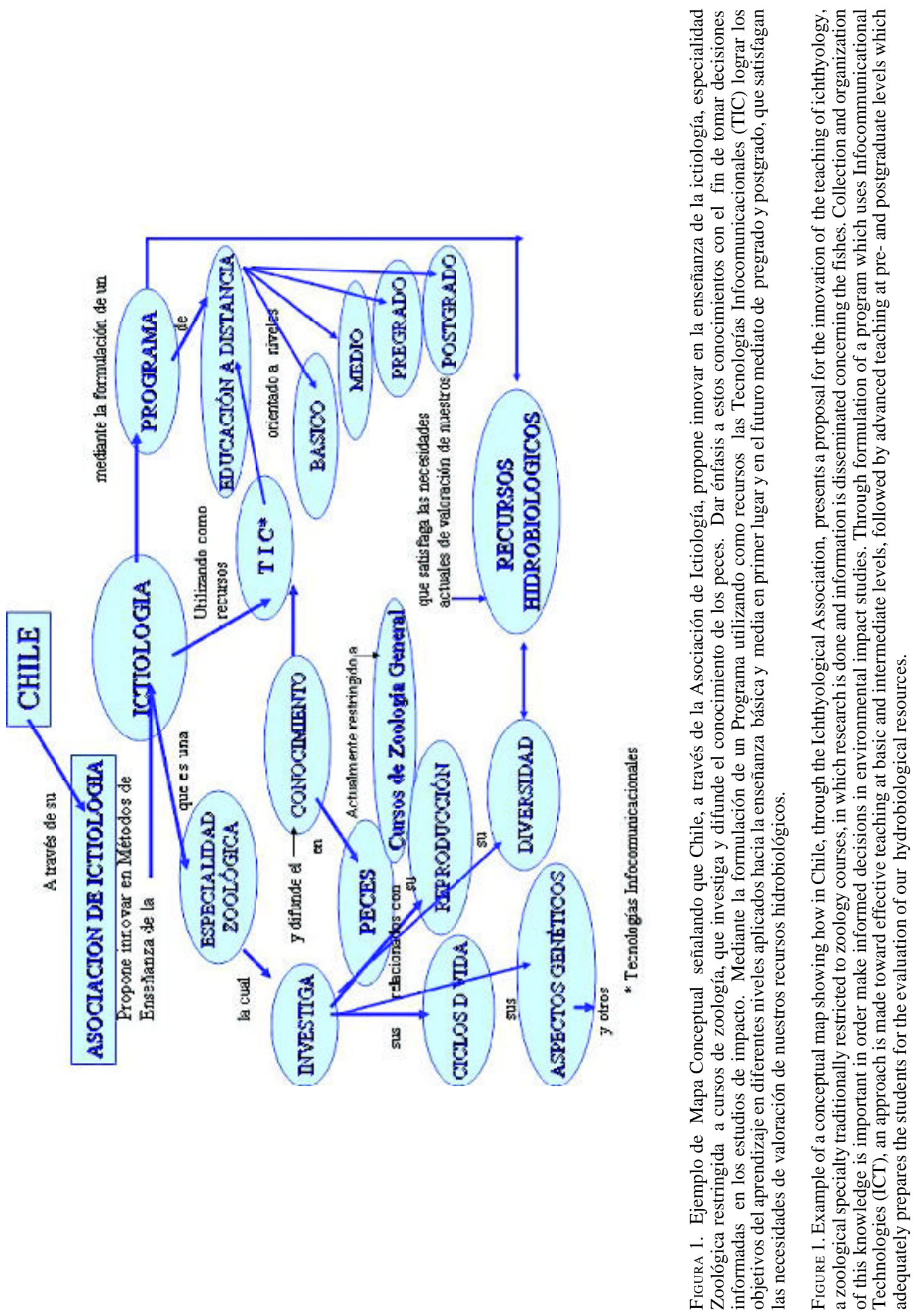
entregar en esta reseña todos los nombres y sus comunicaciones. La resolución de los problemas depende de la cantidad y calidad de la información internalizada (conocimiento) de cada individuo. Se hace así imprescindible el aporte que cada uno pueda entregar en esta gran tarea en cuanto sea asumida.

Este Programa Nacional de enseñanza de la Ictiología trataría de recopilar, almacenar, crear, compartir y utilizar los conocimientos existentes y los nuevos que se vayan incorporando a este gran taller. Habría confidencialidad con respecto al uso por niveles. Nuestro programa debería tener una clave especial conocida por el grupo de los integrantes académicos. Los cursos podrían tener claves específicas de entrada con matrículas a través de las diferentes universidades e instituciones que apoyen el programa.

\section{Preguntas Que SURgen}

- ¿Es de nuestra incumbencia plantear los contenidos e información de la ictiología en Chile?

- ¿Cómo podemos despertar la necesidad de aprendizaje en estas temáticas?

-¿Qué aspectos y cuáles son los enfoques de la ictiología que deberían conocer en forma básica la población en Chile?

-¿Qué información básica debería tener un programa de ictiología para educación a distancia?

- ¿Podemos y o deberíamos adecuar los contenidos a las necesidades e intereses de la piscicultura actual?

\section{OBJetivo General}

1. Sobre la base de los cambios de la enseñanza, modelos conceptuales, uso de redes computacionales e interacción con retroalimentación, llegar a la población que tenga interés, abarcar un mayor número de personas, profesores de enseñanza media, enseñanza básica, alumnos de pre y postgrado de las universidades y en general entregar información ictiológica por medio del uso de esta nueva tecnología en la enseñanza de este grupo importante de recursos biológicos de Chile.

2. Entregar, por medio de la red Internet, información actualizada de temas ictiológicos en cantidad y calidad determinada por niveles (básico, medio, pre-grado, postgrado).

\section{OBJETIVOS ESPECÍfICOS}

1. Motivar a las personas que entren a estos cursos en la búsqueda de información ictiológica a través de programas de diferentes niveles. Clases preparadas o temas desarrollados con algunos objetivos concretos podrían proponerse en temas específicos.

2. Desarrollar el interés por conocer los grandes grupos de recursos ictiológicos existentes en el mundo y en Chile.

3. Conocer y definir ciclos de vida, hábitat y adaptaciones de especies. Analizar y comprender aspectos morfofuncionales.

4. Registrar, analizar y clasificar peces de interés comercial de Chile

5. Crear, almacenar y compartir conocimientos para utilizarlos en esta nueva dimensión, con espacios actualizados en tiempos definidos.

6. Propender al uso de mapas conceptuales, los que pueden ayudar en forma importante tanto a la organización como al uso del conocimiento.

7. Colaborar en la resolución de problemas que competen a la ictiología por integrantes académicos y profesionales integrados de las diferentes universidades e instituciones que apoyen el programa, participantes de la red.

\section{METODOLOGIAS}

1. Uso de nueva tecnología aplicada a la docencia

2. Correo Electrónico

3. Montaje de cursos en Internet

4. Manejo de estadísticas pesqueras: FAO, SERNAP, Banco Central.

5. Recursos pedagógicos con CD o paquetes de diapositivos que puedan aplicarse a diferentes cursos

6. Video-conferencias entre Instituciones Asociadas.

7. Otros recursos técnicos (CD, Videos)

DesarRollo INICIAL CON LA PROPOSICIÓN DE CONTENIDOS PROGRAMÁTICOS

Buscando información sobre los peces de Chile.

I. ¿Cómo, en qué momento y cuál sería el centro de origen los primeros peces sobre el planeta? (de origen filogenético de los peces.) 
Ictiología en la enseñanza básica y media en Chile: HuAQuín, L.

Contenidos en: series de tiempo de la historia de la tierra.

- Aparición de las primeras formas de vida. Eslabones entre invertebrados y vertebrados (Cephalochordata, Urochordata).

- Peces flecha y piures, ¿cuál es su parentesco?.

Entorno posible para la aparición de los primeros peces. Ostracodermos (grandes peces armados).

- Series evolutivas, Placodermos. Peces actuales.

II. ¿Cuáles son los peces actuales que se encuentran en océanos, mares y aguas interiores del planeta?

Grupos de peces sin mandíbulas (Agnatha): Ciclóstomos

¿Qué representantes tenemos en Chile?

Peces mandibulados (Gnatosthomata): Cartilaginosos (Chondrichthyes)

"El fascinante mundo de los esqualos" (título de seminario de una estudiante).

Peces Oseos (Osteichthyes) de Chile

III. ¿Cómo se reproducen y desarrollan?

(Origen ontogenético de los peces). El desarrollo del pejerrey de río (Basilichthys australis).

Aspectos reproductivos, maduración de gametos (ovocitos, espermatozoides) especificidad de cubiertas ovocitarias.

Fecundación (externa-interna). Formación del cigoto, tipo de segmentación. Desarrollo embrionario hasta la eclosión.

IV. ¿A qué obedece la forma, simetría, anatomía y aspectos morfológicos diversos de los peces?.

Análisis morfológico por grupos en peces actuales. Características comunes (cuerda dorsal o notocorda, sistema nervioso, hendiduras faríngeas).

Aspectos diferenciales de peces primitivos Agnatha Ciclostomata:

Mixines y Cefalaspidomorfos. Peces modernos: Gnathostomata, (Condrichthyes, Osteichthyes)

Morfología externa: Cabeza, boca, órganos de los sentidos, hendiduras branquiales, opérculo tronco, cola, aletas impares, pares, cubierta corporal, piel, escamas.

Morfología interna: sistemas tegumentario, muscular, esquelético, digestivo, circulatorio, respiratorio, renal, nervioso, reproductivo.
V. Diversidad de la fauna íctica de Chile en relación con los peces del mundo. $\mathrm{N}^{\mathrm{o}}$ relativo de especies de peces del planeta y en Chile.

Peces en ambientes acuáticos marinos, estuariales y continentales.

Condiciones físico-químicas relacionadas con la longitud y latitud

Cómo afectan los cambios de temperatura (estenotermos, euritermos).

Peces de aguas cálidas y peces de aguas frías Peces de fondo, demersales, pelágicos.

VI. Océanos del mundo y su fauna íctica, Antártida, aguas continentales

\section{Niveles tróficos}

Peces de trofía primaria, secundaria, terciaria

VII. Parasitismo: Peces parásitos y parasitados.

VIII.Los peces y el hombre

Capturas mundiales, aprovechamiento de sus productos

Cultivos. Especies cultivadas

Son ocho grandes temáticas o más las que podrían proponerse y cada una de ellas podría ser tratada para diferentes niveles en la educación, como talleres o cursos modulares.

\section{CONCLUSION}

Los cambios acelerados en las últimas décadas nos están transportando a nuevas formas de educación, de búsqueda de información, de entrega de conocimientos, de relaciones interpersonales El gran objetivo de esta proposición es trascender los aspectos educativos tradicionales y formales. Activar una programación de cursos novedosos a diferentes niveles aplicando tecnología moderna, con muchas imágenes interesantes, colocar nuestras mejores experiencias y nuestro trabajo al servicio de la comunidad estudiosa en temas de la Ictiología. Como valor agregado tendremos una mayor unión entre los especialistas, una Asociación más fuerte por el hecho de mantenernos informados y actualizados con miras a realizar proyectos en conjunto. Para ello, hay numerosas formas de 
proteger la autoría, la academia y las instituciones involucradas.

No es fácil plantear una proposición así, pues involucra compromiso y mucho esfuerzo, sin embargo se plantea porque es una fuerte inquietud la protección de los recursos hidrobiológicos. En los sistemas límnicos como lo señalan Oyarzún y Melendez (2002) hay una inquietud de los ictiólogos que tienen una imperiosa necesidad de conocer y contribuir a salvaguardar la pobre diversidad de los peces dulceacuícolas de Chile, por ser cada día mayor la intervención en sus hábitats. Por lo demás existe un compromiso con la educación de este país, que muchos investigadores y profesores en estas temáticas han planteado.

\section{AGRADECIMIENTOS}

A la profesora Clara Braghiroli (ex catedrática de Física, investigadora educativa y capacitadora docente en la Facultad de Ingeniería de la Universidad de Buenos Aires Rep. Argentina) por sus valiosas sugerencias a la figura del mapa conceptual.

\section{BIBLIOGRAFIA}

Astudillo, A. \& M.T. LóPEZ. 1987. Consideraciones acerca de una nueva educación del medio rural. I Seminario de Desarrollo Sostenido. Una estrategia de Desarrollo Rural. 85-86.

Burbidge, P. 1995. Practical methods for conserving biodiversity. Bradstock R.A. etal. (ed) Australia p $11-36$.
Ministerio de EduCACión. 1996 Objetivos fundamentales y contenidos mínimos de la Educación Básica chilena. Ministerio de Educación (Mineduc). Santiago, Chile.

CRACRAFT, J. 1995. The urgency of building global capacity for biodiversity science. Biodiversity and Conservation 4 (5): 463-475.

Greca, I.M. \& M.A. Moreira. 1998. Modelos mentales, Modelos Conceptuales y Modelización. Cuad. Cut. Ens. Ph. 18 (2):107- 128.

Huaquín, L. \& A. Manríquez. 1986. Problemática y perspectivas de los peces nativos de aguas continentales chilenas. Centro de investigación y planificación del medio ambiente (CIPMA). Segundo encuentro científico sobre el medio ambiente. Tomo I: 132-138.

López, M.T. \& E. Monsalve. 1994. Educación, fundamento para la gestión ambiental y el desarrollo sostenido. Comun. Mus. Reg. Concepción 2: 43-46.

NovaK, J.D. 1990. Concepts maps and Vee diagrams: two metacognitive tools to facilitate meaningful learning. Instructional Science 19: 29-52.

OyARZúN, C. \& R. M ELÉNDEZ. 2002. ¿Qué está pasando con la Ictiología en Chile hoy? Gayana 66 (2): 180.

Pequeño, G. 1989. Peces de Chile. Lista Sistemática revisada y comentada. Rev. Biol. Mar. Valparaíso, 24(2): 1-132.

Ruiz, V.H. \& M. Marchant. 2004. Ictiofauna de aguas continentales chilenas. Universidad de Concepción, Chile. Proyecto de Docencia $\mathrm{N}^{\circ} 98$ $071,356 \mathrm{p}$.

Sernapesca. 2002. Anuario estadístico de pesca 2002. Servicio Nacional de Pesca. Ministerio de Economía Fomento y Reconstrucción Chile.p. 151.

Vila, I., L. Fuentes \& M. Contreras. 1999. Peces Límnicos de Chile. Boletín del Museo Nacional de Historia Natural, Chile, 48: 61-75.

Young, L., J.Stuardo \& I. S.F.Jones. 2002. "Educating the next generation of Oceanographers" in regional schools on the culture and international language of science. Gayana 66(2): 141-146. 